\title{
INVESTMENT ANALYTICS
}

\section{IN THE DAWN OF}

ARTIFICIAL INTELLIGENCE

\section{Combining Upside with Black Swan Scenarios}

\subsection{Use Case}

Portfolio manager Jack manages an institutional fund of $\$ 150$ million. The fund has no explicit liability profile to match and does not contain any investment with a near-term maturity date. Some of its holdings do not have daily liquidity, but historically only a minor percentage of its holdings may need to be withdrawn so as to support the cash needs of its sponsors. Hence, its investments cannot be wholly illiquid. The strategic allocation of the fund has been modeled by an investment consultant as an Asian High-Networth portfolio in Figure 12.1, as follows:

\begin{tabular}{|c|c|c|c|c|c|c|c|}
\hline \multicolumn{2}{|l|}{ Description } & \multirow{2}{*}{$\begin{array}{c}\text { Position in USD } \\
\text { Actual }\end{array}$} & \multirow{2}{*}{$\begin{array}{l}\text { Weight } \\
\text { Actual }\end{array}$} & \multirow{2}{*}{$\begin{array}{l}\text { Return } \\
\text { Historica... }\end{array}$} & \multicolumn{3}{|c|}{ Chinese and Global Economies Diverge } \\
\hline Product - & Asset Class & & & & Pos Chg & Asst Chg & Scen Prc \\
\hline Ascendas Real Estate Investment Tr... & Equity & $999,850.21$ & $6.62 \%$ & $7.44 \%$ & $0.17 \%$ & $2.50 \%$ & 2.74 \\
\hline DBS Group Holdings Limited & Equity & $536,436.49$ & $3.55 \%$ & $49.92 \%$ & $0.72 \%$ & $20.25 \%$ & 34.45 \\
\hline Genting Singapore PLC & Equity & $566,207.31$ & $3.75 \%$ & $28.86 \%$ & $0.45 \%$ & $11.95 \%$ & 1.41 \\
\hline Keppel Corporation Limited & Equity & $225,584.18$ & $1.49 \%$ & $34.08 \%$ & $0.00 \%$ & $-0.25 \%$ & 7.51 \\
\hline Keppel REIT Management Limited & Equity & $602,905.93$ & $3.99 \%$ & $11.81 \%$ & $0.94 \%$ & $23.63 \%$ & 1.42 \\
\hline PIMCO 0-5 Year Hi Yld Corp Bond Id... & Fixed Inco... & $1,000,700.00$ & $6.63 \%$ & $-0.20 \%$ & $0.48 \%$ & $7.22 \%$ & 107.30 \\
\hline PowerShares Golden Dragon China & Equity & $1,754,550.00$ & $11.62 \%$ & $27.53 \%$ & $7.41 \%$ & $63.73 \%$ & 82.08 \\
\hline SPDR Barclays Aggregate Bond ETF & Equity & $832,500.00$ & $5.51 \%$ & $4,380.32 \ldots$ & $-0.16 \%$ & $-2.98 \%$ & 26.92 \\
\hline SPDR Gold Shares & Commodity & $2,464,600.00$ & $16.33 \%$ & $2.64 \%$ & $-6.35 \%$ & $-38.90 \%$ & 75.30 \\
\hline Straits Times Index ETF & Equity & $788,645.90$ & $5.22 \%$ & $20.01 \%$ & $0.53 \%$ & $10.17 \%$ & 3.87 \\
\hline Vanguard MSCI Pacific ETF & Equity & $1,109,400.00$ & $7.35 \%$ & $10.64 \%$ & $2.62 \%$ & $35.66 \%$ & 100.33 \\
\hline iPath S\&P GSCI Crude Oil TR Index ... & Commodity & $7,500.00$ & $0.05 \%$ & $-33.00 \%$ & $0.01 \%$ & $18.12 \%$ & 0.18 \\
\hline iShares Core S\&P500 ETF & Equity & $4,207,650.00$ & $27.87 \%$ & $11.33 \%$ & $9.89 \%$ & $35.50 \%$ & 380.09 \\
\hline Total & & $15,096,530.02$ & $100.00 \%$ & $254.47 \%$ & $16.70 \%$ & & \\
\hline
\end{tabular}

Figure 12.1: Asian High Networth Portfolio 
Jack likes this allocation, which has enjoyed an exceptional last year, but simply allocating to the same asset buckets going forward is unlikely to achieve the same desirable results. Jack's goal is to generate a steady absolute return over the medium to the long term. His asset sponsors are well aware of that markets may swing up and down in the shorter term, and that artificially creating an absolute return vehicle may be very costly and hurt returns without achieving the sponsor's longer-term investment objectives.

Jack's challenge is to allocate to "good" sectors while maintaining some level of confidence that his portfolio will not be hurt by a massive drawdown later. His asset sponsors are prepared to accept such drawdowns assuming that the portfolio upside will be more than sufficient to pay for any potential downside. Hence, his asset sponsors demand to "trust but verify": They would like to see a sophisticated analysis that Jack's bets stand reasonable chances of paying off.

This chapter will show Jack how to achieve such a feat systematically.

\subsubsection{Defining the Investment Problem}

To achieve his complex investment goal, Jack faces several significant limitations associated with the characteristics of any multi-asset portfolio.

First, it is common knowledge among professional investors and proven by empirical research commissioned by Norges Bank Investment Management ${ }^{1}$ that most institutional-sized portfolios derive their returns from beta instead of alpha due to their massive size. Intuitively, it is difficult for any large institution at a size comparable to a small financial market to outperform any one specific market: They will buy "up" the market on the way in and drive the market "down" on exit.

Second, any significant change to the portfolio will result in high transaction costs due to market impact, which will offset the benefits of trying to achieve meaningful returns. Further, hedging may not be a practical strategy for a large portfolio as such, because the massive size of any short positions required by an effective hedge may artificially induce a market crash.

Finally, there are always some investments in any portfolio that may be impossible to sell en masse, such as real estate investments.

After the financial crisis in 2008, the sponsors of Jack's fund modified his investment mandate to account for potential "Black Swan" scenarios in its routine portfolio rebalancing reviews, because it may be impossible to make any sudden,

${ }^{1} 20$ January 2014, Review of the Active Management of the Norwegian Government Pension Fund Global, https://www0.gsb.columbia.edu/faculty/aang/papers/ AngBrandtDenison.pdf. 
major changes to such a large portfolio or put on any hedge even with, say, 6 months of visibility that such a "Black Swan" event does happen. Yet, such a fund will not generate a healthy medium to long-term return if it is "permanently" positioned to avoid extreme events. Jack wants to find an optimal asset allocation that can take into account low-probability extreme events for a multi-asset portfolio.

\subsubsection{Potential Scenarios on Watch}

Back in 2014, China's domestic market was denied entry into internationallywatched MSCI's index benchmark for the third year in a row because the level of freedom in the buying and selling of China A shares was deemed insufficient for international investors to freely invest and divest in the view of MSCI. This rejection frustrated certain investors and was expected to serve as one way to motivate the Chinese government to take more aggressive actions towards financial market liberation. Therefore, Jack held the view that the speed at which the Chinese market would open up to foreign investors soon was going to accelerate, which he described as "Aggressive Chinese Market Liberation". Concurrently, the Middle East was not doing well due to the collapse of oil prices since the beginning of 2014. Jack believes that the Middle East would not recover soon, but might even slow down its growth, which he describes as "Middle East Deteriorates". Due to the increase in interest rate in the US, the dollar was getting stronger. However, Jack believed CNY would keep increasing its value once the Chinese government took action in the foreign exchange market, which he called "Chinese and Global Economies Diverge".

\subsubsection{Traditional Approach}

Traditionally, Jack would start with the classic approach of maximizing portfolio Sharpe Ratio. He first obtains the expected return of each asset under each scenario. From those expected returns, he calculates the optimal asset allocation under each scenario (See Figure 12.2). The key disadvantages of this approach are: a) there is no way to reflect the confidence level of each scenario; and b) transaction costs will be potentially prohibitive each time he changes his views. For instance, the Treynor-Black approach as described by [Treynor 1973] assumes that an analyst can make perfect forecasts on future alpha and allocate portfolio weights. Perfect foresight is at best an unrealistic assumption that only results in corner solutions and frequent and expensive switching of asset positions when forecasts change. 


\begin{tabular}{|c|c|c|c|c|c|c|c|c|c|c|c|c|c|}
\hline \multicolumn{2}{|l|}{ Description } & \multirow{2}{*}{$\begin{array}{l}\text { Position in USD } \\
\text { Actual }\end{array}$} & \multirow{2}{*}{$\begin{array}{l}\text { Weight } \\
\text { Actual }\end{array}$} & \multirow{2}{*}{$\begin{array}{c}\text { Return } \\
\text { Historica. }\end{array}$} & \multicolumn{3}{|c|}{ Aggressive Chinese Market Liberalization } & \multicolumn{3}{|c|}{ Middle East Deteriorates } & \multicolumn{3}{|c|}{ Chinese and Global Economies Diverge } \\
\hline Product - & Asset Class & & & & Pos Chg & Asst Chg & Scen Prc & Pos Chg & Asst Chg & Scen Prc & Pos Chg & Asst Chg & Scen Prc \\
\hline PowerShares Golden Dragon China & Equity & $1,754,550.00$ & $11.62 \%$ & $27.53 \%$ & $6.72 \%$ & $57.79 \%$ & 79.10 & $5.70 \%$ & $49.05 \%$ & 74.72 & $7.41 \%$ & $63.73 \%$ & 82.08 \\
\hline SPDR Barclays Aggregate Bond ETF & Equity & $832,500.00$ & $5.51 \%$ & 4,380.32... & $0.91 \%$ & $16.59 \%$ & 32.35 & $-0.16 \%$ & $-2.96 \%$ & 26.93 & $-0.16 \%$ & $-2.98 \%$ & 26.92 \\
\hline SPDR Gold Shares & Commodity & $2,464,600.00$ & $16.33 \%$ & $2.64 \%$ & $0.38 \%$ & $2.30 \%$ & 126.06 & $-1.25 \%$ & $-7.64 \%$ & 113.82 & $-6.35 \%$ & $-38.90 \%$ & 75.30 \\
\hline Straits Times Index ETF & Equity & $788,645.90$ & $5.22 \%$ & $20.01 \%$ & $3.71 \%$ & $70.97 \%$ & 6.00 & $-1.32 \%$ & $-25.27 \%$ & 2.62 & $0.53 \%$ & $10.17 \%$ & 3.87 \\
\hline Vanguard MSCI Pacific ETF & Equity & $1,109,400.00$ & $7.35 \%$ & $10.64 \%$ & $9.41 \%$ & $128.09 \%$ & 168.69 & $-1.75 \%$ & $-23.76 \%$ & 56.39 & $2.62 \%$ & $35.66 \%$ & 100.33 \\
\hline iPath S\&P GSCI Crude Oil TR Index ... & Commodity & $7,500.00$ & $0.05 \%$ & $-33.00 \%$ & $0.03 \%$ & $69.95 \%$ & 0.25 & $0.02 \%$ & $38.14 \%$ & 0.21 & $0.01 \%$ & $18.12 \%$ & 0.18 \\
\hline ishares Core S\&P500 ETF & Equity & $4,207,650.00$ & $27.87 \%$ & $11.33 \%$ & $23.13 \%$ & $83.00 \%$ & 513.33 & $-1.01 \%$ & $-3.63 \%$ & 270.32 & $9.89 \%$ & $35.50 \%$ & 380.09 \\
\hline Total & & $15,096,530.02$ & $100.00 \%$ & $254.47 \%$ & $67.30 \%$ & & & $-7,03 \%$ & & & $16.70 \%$ & & \\
\hline
\end{tabular}

Figure 12.2: Portfolio under Different Scenarios

Jack is also concerned about several practical considerations before choosing a specific implementation:

1. If the portfolio is structured on any specific scenario, it may result in a "corner solution" concentrated in a few assets, rather than a full range of diversified assets. Rebalancing from one corner solution to the next (after a change in investment view) will result in another major change in weights and therefore significant transaction costs; this approach may not be practical for large institutional portfolios because no one knows exactly which scenario may happen, and even their confidence may change.

2. The existing market views should be medium to long-term so as to be consistent with the portfolio's investment goal. Otherwise, the high transaction costs due to dramatic moves in positions even with slight changes in forwardlooking scenarios will exceed any benefits from such portfolio rebalancing.

3. The rebalancing should be done with evidence of appropriate trade-offs between risks and return.

\subsubsection{Stochastic Analysis Solution}

To address the shortcomings of the single scenario approach as discussed above, Jack is going to try a multi-scenario analysis to obtain an optimal asset allocation that will improve under multiple views. The Stochastic Analysis tool allows Jack to input any number of views with the probability of each occurring assigned by Jack. Each view can describe the expected return of a portfolio under each scenario, Thus, such a model combines the three views that Jack holds (i.e., Figure 12.3 "Aggressive Chinese Market Liberation" View, Figure 12.4 "Middle East Deteriorates" View, and Figure 12.5 "Chinese and Global Economies Diverge" View), producing both the set of expected returns of assets and the optimal portfolio weights. Jack assigns his confidence level to each scenario $(10 \%, 10 \%$, and $80 \%$ respectively), which tilts the neutral weights of the portfolio in the direction following his beliefs. These views are shown as follows: 


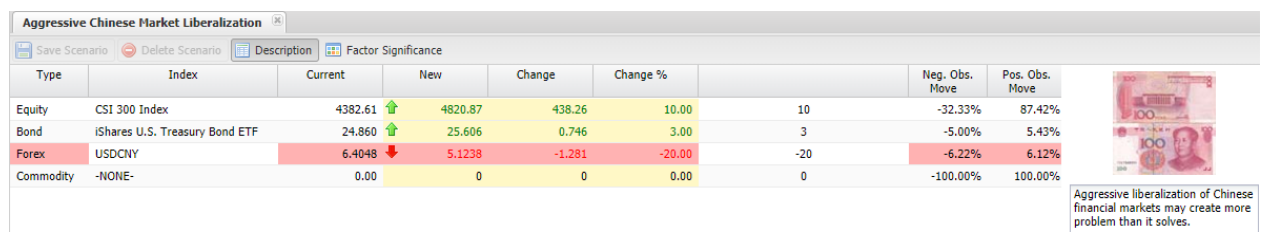

Figure 12.3: The "Aggressive Chinese Market Liberation” View

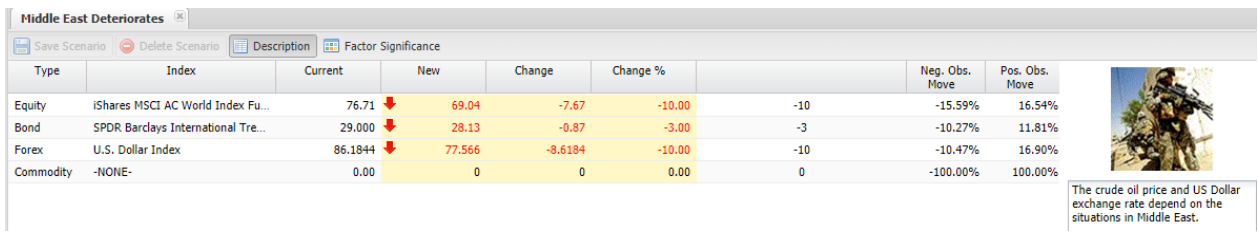

Figure 12.4: The "Middle East Deteriorates" View

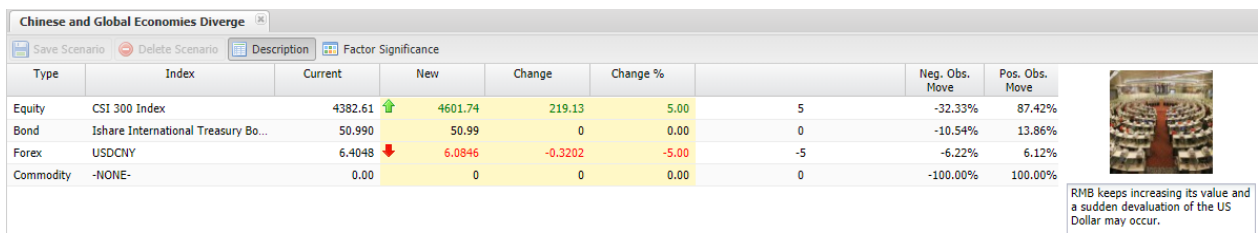

Figure 12.5: The "Chinese and Global Economies Diverge" View

Besides overcoming all the challenges mentioned above in the traditional singlescenario approach, the stochastic analysis approach provides a solution that is intuitive, highly-diversified and less sensitive to small changes in a single input parameter. It also provides an accurate estimation of error maximization. To access the Stochastic Analysis tool, users can click "Analytics" and then look for "Decision/Execution". Users then click "Stochastic Analysis", which is immediately below "User Scenarios".

\subsubsection{Outcome}

Jack is happy to see that this alternative approach allows him to incorporate lowprobability extreme events to construct a more stable portfolio. This recommendation gives Jack a more effective way to pursue his medium-to-long term investment 


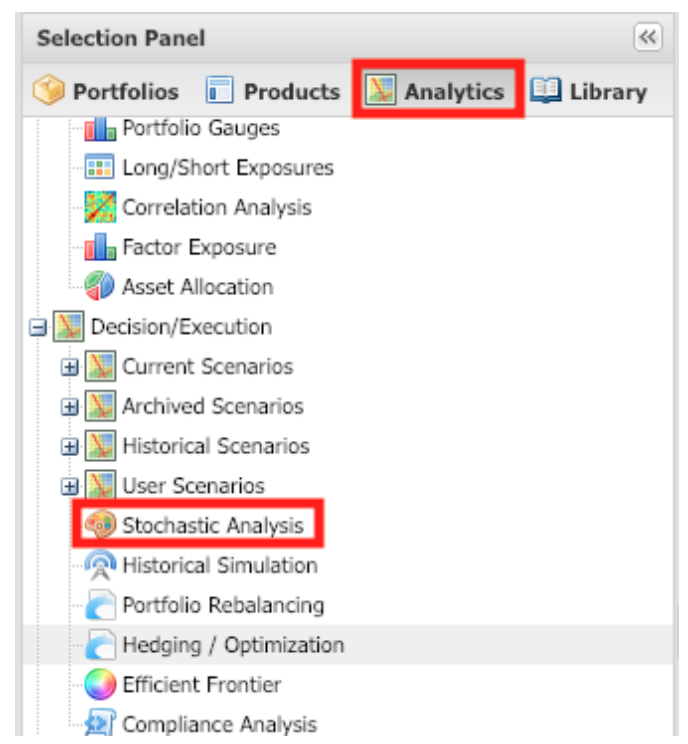

Figure 12.6: "Analytics $\rightarrow$ Stochastic Analysis"

thesis, with inexpensive "protection" against extreme downside outcomes that he does not need to pay option premia for, or comes with costs of carry that add up over time. Given that institutional portfolios tend to be very expensive to rebalance dramatically due to their massive size, now Jack has a scientific way to test his investment ideas under several scenarios simultaneously and get them right prior to implementation.

\subsection{Methodology}

\subsubsection{Objective}

The Stochastic Analysis tool allows investors to combine their views of different market scenarios with different confidence levels so as to achieve a better asset allocation. Through this analysis, a portfolio can achieve both a superior ex-ante upside and is in a better position to mitigate its downside risk. As one example, this tool can be used by investment professionals who want to specify a dominant view, but are also concerned about the potential impact of so-called "Black Swan" events in the market. Stochastic Analysis provides a practical way by which our investors can balance potential gains on the upside against protection from extreme downside scenarios. 


\subsubsection{Overview}

Stochastic Analysis uses a Bayesian approach to combine the subjective views of an investor (the prior distribution) on asset returns with the market equilibrium vector of expected returns, so as to form a combined estimate (the posterior distribution) of expected returns. Basically, Stochastic Analysis can be thought of graphically as shown in Figure 12.7.

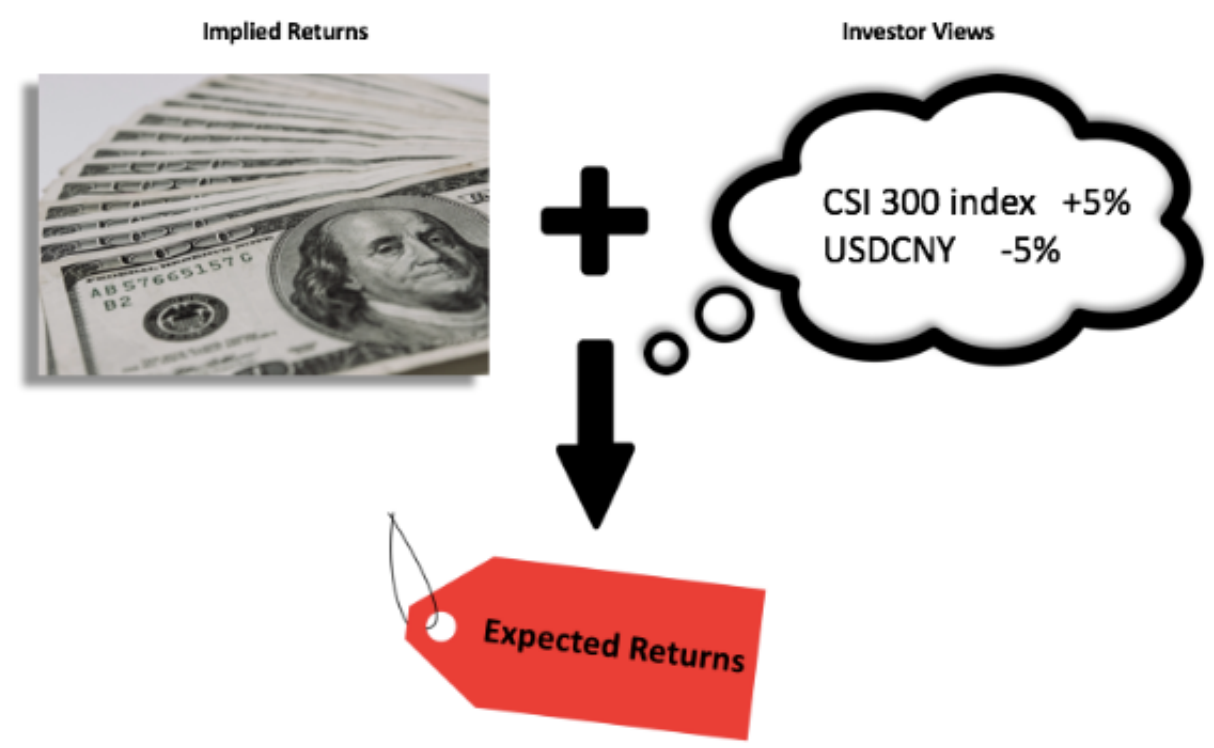

Figure 12.7: Combining Implied Returns and Investor Views

\subsubsection{Formula}

Notations:

$\Pi$ Implied Return, which closed-form solution is provided by $\frac{\Sigma w_{m k t} \cdot\left(\mu^{T} w_{m k t}\right)}{w_{m k t}^{T} \Sigma w_{m k t}}$ without position constraints, representing the return each asset must deliver so as to justify the amount of risk it contributes to the overall portfolio;

$\Sigma$ Covariance matrix of asset returns;

$\mu$ Asset historical return;

$\tau$ A scalar for the investor to control the maximal change(s) of portfolio position(s), which lacks a simple intuitive explanation ${ }^{2}$;

\footnotetext{
${ }^{2}$ See, for instance, A Discussion of Tau, http: / / www . blacklitterman. org/tau . html.
} 
$P$ Matrix representing an investor's view, with each column of matrix $P$ representing an investor's view about the assets' weighted expected returns, and the normalized elements of the columns summing up to 1 ;

$Q$ A column vector, in which each element is a total change of a portfolio's weighted expected returns under a particular view;

$\Omega$ Diagonal covariance matrix of errors terms expressing the uncertainty in each view;

$I$ Identity matrix;

The Stochastic Analysis algorithm, as modified from the classic Black-Litterman model, is given by:

$$
E(R)=\Pi+\tau \Sigma P^{T}\left[\Omega+\tau P \Sigma P^{T}\right]^{-1}(Q-P \Pi)
$$

The key improvement over the classic Black-Litterman model is that the current portfolio weight $\left(w_{m k t}\right)$ can be used as a sufficiently good estimator to the equilibrium market weight. There is no loss of generality from this feature because an investor who is interested in the classic Black-Litterman analysis can simply enter the equilibrium market weight as the current portfolio weight. The optimal asset weight is calculated by the equation:

$$
w_{o p t}=\frac{\Sigma^{-1} E(R)}{I^{T} \Sigma^{-1} E(R)}
$$

\subsubsection{Computational Process}

A typical computation is done in the following 5 steps:

Step 1 Calculate the mark-to-market valuation, and the weights of the existing portfolio;

Step 2 Do a reversed optimization of the Sharpe Ratio to get the Implied Returns of the portfolio assets;

Step 3 Define the scenarios and the confidence or probability of each scenario;

Step 4 Estimate the scenario return of each asset to compute the $P$ matrix and $Q$ vector; and

Step 5 Compute the new optimal weights, by re-optimizing Sharpe Ratio, combining the implied equilibrium distribution and the view distribution.

Choosing the portfolio in Step 1 will lead to the results of Stochastic Analysis in the following schematic: 


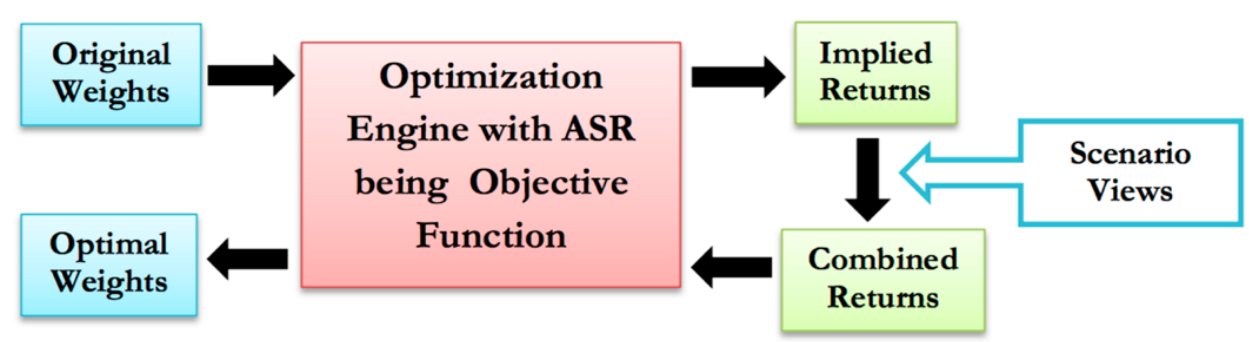

Figure 12.8: Stochastic Analysis Computational Process

The computation process on HedgeSPA platform corresponds to the following five steps of calculations (that can be verified against similar calculations performed in typical statistical packages such as R):

Step 1 Refresh portfolio weights

Compute $w_{m k t}$ of the current portfolio using the latest mark-to-market values of assets.

Step 2 Compute implied returns $\Pi$

Given $w_{m k t}$ of the current portfolio, use reversed optimization to find the vector of Implied Returns $\Pi$, such that those implied returns maximize $\frac{E\left(R_{a}-R_{r f}\right)}{\sqrt{\operatorname{Var}\left(R_{a}-R_{r f}\right)}}$ based on existing portfolio weights, where $R_{a}$ is the portfolio return and $R_{r f}$ is the risk-free return. The close-form solution for the reversed optimization problem without position constraint is $\Pi=\frac{\Sigma w_{m k t} \cdot\left(\mu^{T} w_{m k t}\right)}{w_{m k t}^{T} \Sigma w_{m k t}}$.

Step 3 Compute $P$ matrix

Each column of the $P$ matrix represents a view according to the asset-weighted expected returns. The elements in each column are scaled so that they sum up to $100 \%$.

Step 4 Calculate the combined returns $E(R)$ from the implied returns and forward looking view $E(R)=\Pi+\tau \Sigma P^{T}\left[\Omega+\tau P \Sigma P^{T}\right]^{-1}(Q-P \Pi)$.

Step 5 Given $E(R)$ as computed above, find the optimal allocation of new asset weights to maximize $\frac{E\left(R_{a}-R_{r f}\right)}{\sqrt{\operatorname{Var}\left(R_{a}-R_{r f}\right)}}$. We may solve the problem numerically to account for constraints and non-negative positions, but the result should be similar to the following closed-form solution: $w_{\text {opt }}=\frac{\Sigma^{-1} E(R)}{I^{T} \Sigma^{-1} E(R)}$.

It is not required to use the traditional Sharpe Ratio for this calculation. For instance, a possible alternative is to use the Alternative Sharpe Ratio introduced earlier, even though such a ratio is significantly more computationally intensive to perform both the reversed optimization and the subsequent forward optimization. 


\subsection{Worked Example}

\subsubsection{Overview}

As mentioned earlier, the strategic allocation of Jack's fund can be modeled by an Asian High Net-worth portfolio, which produces a modest historical portfolio return of $1.39 \%$ in Figure 12.9 below. Jack's goal is to generate a steady absolute return over the medium to the long term. The asset sponsor is well aware of that markets may swing up and down in the shorter term, and that artificially creating an absolute return vehicle may be costly and hurt returns without achieving the sponsor's longer-term investment objectives.

\begin{tabular}{|c|c|c|c|c|c|c|c|}
\hline \multicolumn{2}{|l|}{ Description } & \multirow{2}{*}{$\begin{array}{c}\text { Position in USD } \\
\text { Actual }\end{array}$} & \multirow{2}{*}{$\begin{array}{l}\text { Weight } \\
\text { Actual }\end{array}$} & \multirow{2}{*}{$\begin{array}{l}\text { Return } \\
\text { Historica... }\end{array}$} & \multicolumn{3}{|c|}{ Chinese and Global Economies Diverge } \\
\hline Product - & Asset Class & & & & Pos Chg & Asst Chg & Scen Prc \\
\hline Ascendas Real Estate Investment Tr... & Equity & $999,850.21$ & $6.62 \%$ & $7.44 \%$ & $0.17 \%$ & $2.50 \%$ & 2.74 \\
\hline DBS Group Holdings Limited & Equity & $536,436.49$ & $3.55 \%$ & $49.92 \%$ & $0.72 \%$ & $20.25 \%$ & 34.45 \\
\hline Genting Singapore PLC & Equity & $566,207.31$ & $3.75 \%$ & $28.86 \%$ & $0.45 \%$ & $11.95 \%$ & 1.41 \\
\hline Keppel Corporation Limited & Equity & $225,584.18$ & $1.49 \%$ & $34.08 \%$ & $0.00 \%$ & $-0.25 \%$ & 7.51 \\
\hline Keppel REIT Management Limited & Equity & $602,905.93$ & $3.99 \%$ & $11.81 \%$ & $0.94 \%$ & $23.63 \%$ & 1.42 \\
\hline PIMCO 0-5 Year Hi Yld Corp Bond Id... & Fixed Inco... & $1,000,700.00$ & $6.63 \%$ & $-0.20 \%$ & $0.48 \%$ & $7.22 \%$ & 107.30 \\
\hline PowerShares Golden Dragon China & Equity & $1,754,550.00$ & $11.62 \%$ & $27.53 \%$ & $7.41 \%$ & $63.73 \%$ & 82.08 \\
\hline SPDR Barclays Aggregate Bond ETF & Equity & $832,500.00$ & $5.51 \%$ & $4,380.32 \ldots$ & $-0.16 \%$ & $-2.98 \%$ & 26.92 \\
\hline SPDR Gold Shares & Commodity & $2,464,600.00$ & $16.33 \%$ & $2.64 \%$ & $-6.35 \%$ & $-38.90 \%$ & 75.30 \\
\hline Straits Times Index ETF & Equity & $788,645.90$ & $5.22 \%$ & $20.01 \%$ & $0.53 \%$ & $10.17 \%$ & 3.87 \\
\hline Vanguard MSCI Pacific ETF & Equity & $1,109,400.00$ & $7.35 \%$ & $10.64 \%$ & $2.62 \%$ & $35.66 \%$ & 100.33 \\
\hline iPath S\&P GSCI Crude Oil TR Index ... & Commodity & $7,500.00$ & $0.05 \%$ & $-33.00 \%$ & $0.01 \%$ & $18.12 \%$ & 0.18 \\
\hline iShares Core S\&P500 ETF & Equity & $4,207,650.00$ & $27.87 \%$ & $11.33 \%$ & $9.89 \%$ & $35.50 \%$ & 380.09 \\
\hline Total & & $15,096,530.02$ & $100.00 \%$ & $254.47 \%$ & $16.70 \%$ & & \\
\hline
\end{tabular}

Figure 12.9: The "Asian High Net-Worth" Portfolio

Jack comes up with three possible market outcomes, which he describes as "Chinese and Global Economics Diverge" (in Figure 12.3), "Aggressive Chinese Market Liberalization" (in Figure 12.4), and "Middle East Deterioration" (in Figure 12.5) scenarios with different shocks on his chosen market indices.

\subsubsection{Definitions}

1. Mathematical Models

- Modeling Parameters

a. This worked example uses 4 years of historical data from May 2012 to May 2016. 
b. In Figure 12.9 above, the annual historical return for each asset is calculated using the forward 6-month rolling windows over those 4 years. For consistency, all the statistics are computed over the same horizon, but they may be annualized whenever appropriate.

c. The risk-free rate of return is set to be $0.1 \%$ during this period, and is assumed to be constant.

- Sharpe Ratio

The Sharpe Ratio is given by $\frac{E\left[R_{a}-R_{r f}\right]}{\sqrt{\operatorname{Var}\left[R_{a}-R_{r f}\right]}}$, where $R_{a}$ is the asset return, $R_{r f}$ is the risk-free asset return and risk of the portfolio is measured by the variance of excessive return. The Sharpe Ratio lets an investor know whether a higher return of a portfolio is achieved via better investment decisions (or alpha), or simply from taking excessive risk as measured by volatility (or beta). The greater a portfolio's Sharpe Ratio is, the better its risk-adjusted performance has been, although the Sharpe Ratio does come with some well-known shortcomings over its inability to properly account for portfolio tail risk.

- Portfolio Optimization and Reversed Optimization

In this worked example, portfolio optimization is used to find the optimal asset weight allocation that maximizes/minimizes an objective function under specified constraints (e.g. non-negative weight is a common portfolio constraint), given the expected return of each asset under each scenario. Reversed portfolio optimization calculates the implied return of each asset in the portfolio so that the current weights are considered optimal under a specified choice of objective function.

2. Naïve Modeling

A typical "Naïve Modeling" strategy is to optimize the portfolio by maximizing portfolio Sharpe Ratio under each scenario. Such a model is implemented in two steps:

a. First Step: Calculate the expected returns under each view

The expected return for each asset under each view is calculated by individually running a regression on each asset's returns against the market factor returns under each view in Figure 12.2. The result is displayed in the "Asst Chg" column in Figure 12.10. 
b. Second Step: Calculate the optimal weight allocation

Solve for $w=\left[w_{1}, w_{2}, \cdots w_{13}\right]^{T}$, in order to maximize the objective function $\frac{\left(E\left[R_{a}-R_{r f}\right]\right)}{\sqrt{\left(\operatorname{Var}\left[R_{a}-R_{r f}\right]\right)}}$, subject to:

Constraint 1: $w_{1}+w_{2} \cdots w_{13}=1$

Constraint 2: $w_{i} \geq 0$ for $i=1,2, \cdots, 13$

c. Final Step: Interpret the results from naïve modeling

Figure 12.10 shows the return shocks of the portfolio before optimization:

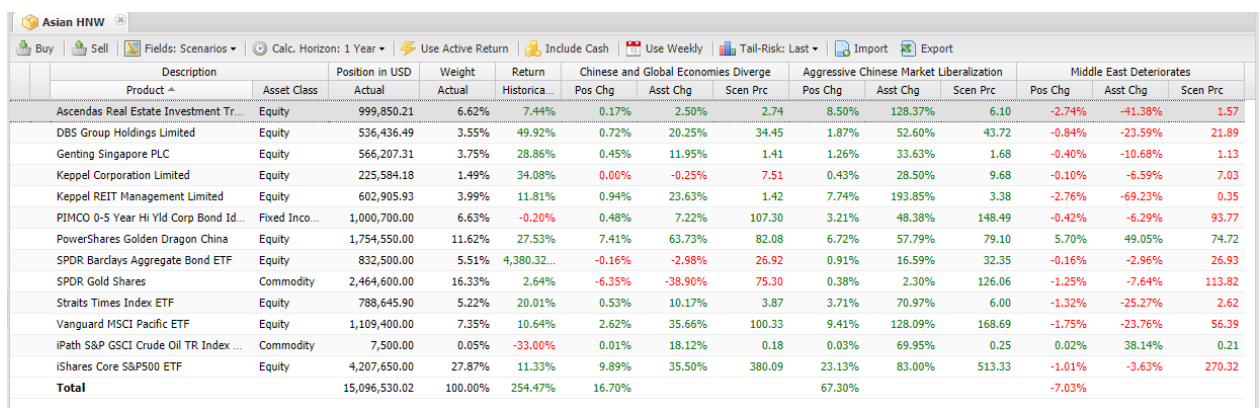

Figure 12.10: Portfolio under the 3 Scenarios before Optimization

Table 12.1: Portfolio Return, Standard Deviation, and Sharp Ratio Optimized Individually Under the 3 Scenarios

\begin{tabular}{|l|r|r|r|r|}
\hline Scenario & Original & Chinese and Global Econ. Diverge & Aggressive Chinese Liberalization & Middle East Deteriorates \\
\hline Portfolio Return & $0.3 \%$ & $53.1 \%$ & $95.1 \%$ & $1.8 \%$ \\
\hline Portfolio Stdev & $6.2 \%$ & $24.4 \%$ & $65.7 \%$ & $29.4 \%$ \\
\hline Sharp Ratio & 0.05 & 2.18 & 1.45 & 0.06 \\
\hline
\end{tabular}

Table 12.1 shows that the portfolio optimized under each of the 3 scenarios ends up with more extreme portfolio returns and volatilities.

These charts and tables illustrate the type of "corner solutions" with very large changes in asset weights, which can rarely be executed by real-life portfolio managers in practice due to high transaction costs.

3. Mathematical Properties of the classic Black-Litterman Model

When the confidence level is $0 \%$ for every view, the optimal weight is given by $w_{M k t}$ and $E[R]=$ Implied Returns. When confidence level is $0 \%$ under the 
Chinese and Global Economies Diverge view, the portfolio return is $1.39 \%$, which corresponds to the original portfolio return in Figure 12.1. When there are no additional views added, the model gives the original asset weight allocation. The results are stated in Table 12.2, as follows:

Table 12.2: Portfolio Weights under 0\% Confidence

\begin{tabular}{|r|r|r|r|}
\hline No. & Assets & Weight & Return \\
\hline 1 & Ascendas Real Estate Investment Trust & $5.66 \%$ & $10.24 \%$ \\
\hline 2 & DBS Group Holdings Limited & $1.87 \%$ & $-36.15 \%$ \\
\hline 3 & Genting Singapore PL & $2.07 \%$ & $-4.57 \%$ \\
\hline 4 & Keppel Corporation Limited & $1.03 \%$ & $-32.59 \%$ \\
\hline 5 & Keppel REIT Management Limited & $3.53 \%$ & $-14.93 \%$ \\
\hline 6 & PIMCO 0-5 Year Hi Yld Corp Bond Idx ETF & $6.23 \%$ & $-4.49 \%$ \\
\hline 7 & PowerShares Golden Dragon China & $6.19 \%$ & $-35.19 \%$ \\
\hline 8 & SPDR Barclays Aggregate Bond ETF & $11.55 \%$ & $7.17 \%$ \\
\hline 9 & SPDR Dow Jones Global Real Estate & $15.91 \%$ & $14.86 \%$ \\
\hline 10 & SPDR Gold Shares & $16.17 \%$ & $22.84 \%$ \\
\hline 11 & Straits Times Index ETF & $4.04 \%$ & $-18.09 \%$ \\
\hline 12 & Vanguard MSCI Pacific ETF & $5.33 \%$ & $-14.38 \%$ \\
\hline 13 & iShares Core S\&P500 ETF & $20.41 \%$ & $-2.38 \%$ \\
\hline & Portfolio Return & $100 \%$ & $1.39 \%$ \\
\hline
\end{tabular}

4. Additional Parameters and calculations about the views used in this worked example

(a) Inputs of Confidence Level and Tau:

Table 12.3: Input parameters of the Stochastic Analysis Model

\begin{tabular}{|l|r|}
\hline Confidence Level & $80 \%$ \\
\hline Minimum Tau & 0.01 \\
\hline Maximum Tau & 0.1 \\
\hline
\end{tabular}


(b) Correlation Matrix : Matrix gives the correlations between all pairs of asset returns.

Table 12.4: Correlation Matrix

$\begin{array}{lrrrrrrrrrrrrr}\text { Assets No. } & \text { No.1 } & \text { No.2 } & \text { No.3 } & \text { No.4 } & \text { No.5 } & \text { No.6 } & \text { No.7 } & \text { No.8 } & \text { No.9 } & \text { No.10 } & \text { No.11 } & \text { No.12 } & \text { No.13 } \\ \text { No.1 } & 0.038 & 0.031 & 0.035 & 0.027 & 0.020 & 0.010 & 0.014 & 0.002 & 0.000 & 0.009 & 0.007 & 0.025 & 0.015 \\ \text { No.2 } & 0.031 & 0.096 & 0.065 & 0.071 & 0.052 & 0.017 & 0.056 & 0.004 & -0.012 & 0.022 & 0.001 & 0.061 & 0.035 \\ \text { No.3 } & 0.035 & 0.065 & 0.098 & 0.075 & 0.039 & 0.016 & 0.054 & 0.004 & -0.005 & 0.024 & -0.001 & 0.053 & 0.032 \\ \text { No.4 } & 0.027 & 0.071 & 0.075 & 0.189 & 0.045 & 0.015 & 0.099 & 0.009 & -0.029 & 0.060 & -0.016 & 0.056 & 0.073 \\ \text { No.5 } & 0.020 & 0.052 & 0.039 & 0.045 & 0.044 & 0.011 & 0.030 & 0.003 & -0.011 & 0.015 & 0.005 & 0.037 & 0.022 \\ \text { No.6 } & 0.010 & 0.017 & 0.016 & 0.015 & 0.011 & 0.006 & 0.009 & 0.001 & 0.000 & 0.004 & 0.003 & 0.013 & 0.007 \\ \text { No.7 } & 0.014 & 0.056 & 0.054 & 0.099 & 0.030 & 0.009 & 0.115 & 0.004 & -0.024 & 0.032 & -0.007 & 0.040 & 0.042 \\ \text { No.8 } & 0.002 & 0.004 & 0.004 & 0.009 & 0.003 & 0.001 & 0.004 & 0.001 & -0.002 & 0.003 & -0.001 & 0.003 & 0.004 \\ \text { No.9 } & 0.000 & -0.012 & -0.005 & -0.029 & -0.011 & 0.000 & -0.024 & -0.002 & 0.033 & -0.008 & 0.009 & -0.008 & -0.015 \\ \text { No.10 } & 0.009 & 0.022 & 0.024 & 0.060 & 0.015 & 0.004 & 0.032 & 0.003 & -0.008 & 0.024 & -0.005 & 0.017 & 0.024 \\ \text { No.11 } & 0.007 & 0.001 & -0.001 & -0.016 & 0.005 & 0.003 & -0.007 & -0.001 & 0.009 & -0.005 & 0.045 & 0.003 & -0.008 \\ \text { No.12 } & 0.025 & 0.061 & 0.053 & 0.056 & 0.037 & 0.013 & 0.040 & 0.003 & -0.008 & 0.017 & 0.003 & 0.046 & 0.026 \\ \text { No.13 } & 0.015 & 0.035 & 0.032 & 0.073 & 0.022 & 0.007 & 0.042 & 0.004 & -0.015 & 0.024 & -0.008 & 0.026 & 0.034\end{array}$

(c) $P$ Matrix, $Q$ Matrix and $\Omega$ Matrix

An intuitive interpretation of the the $P$ Matrix, $Q$ Matrix and $\Omega$ Matrix is paraphrased as follows: ${ }^{3}$

Moving from views to the inputs used in the classic Black-Litterman model can be confusing. First, the model does not specify views on all assets. In the thirteen asset example, the number of views $k$ is 3 ; thus, the View Vector $Q$ is a $3 \times 1$ column vector. The uncertainty of the views is expressed by a normally-distributed error term vector $\varepsilon$ with a mean of 0 and covariance matrix $\Sigma$. Hence, a view is given by $Q+\varepsilon$.

Conceptually, the classic Black-Litterman model is a weighted average of the Implied Return vector $\Pi$ and the View Vector $Q$, in which the relative weightings are computed from the scalar $\tau$ and the uncertainty of the views $\Omega$. Unfortunately, the scalars and the uncertainties in the views are abstract without simple intuitive explanations. If an investor expresses great the confidence in the expressed views, the new return vector will be closer to the expressed views. Alternatively, if the investor is not confident in the expressed views, the new return vector should be closer to the Implied Return vector $\Pi$.

\footnotetext{
${ }^{3}$ Source: see https://faculty. fuqua.duke.edu/ charvey/Teaching/BA453 $2006 /$ Idzorek_onBL.pdf for a complete explanation.
} 


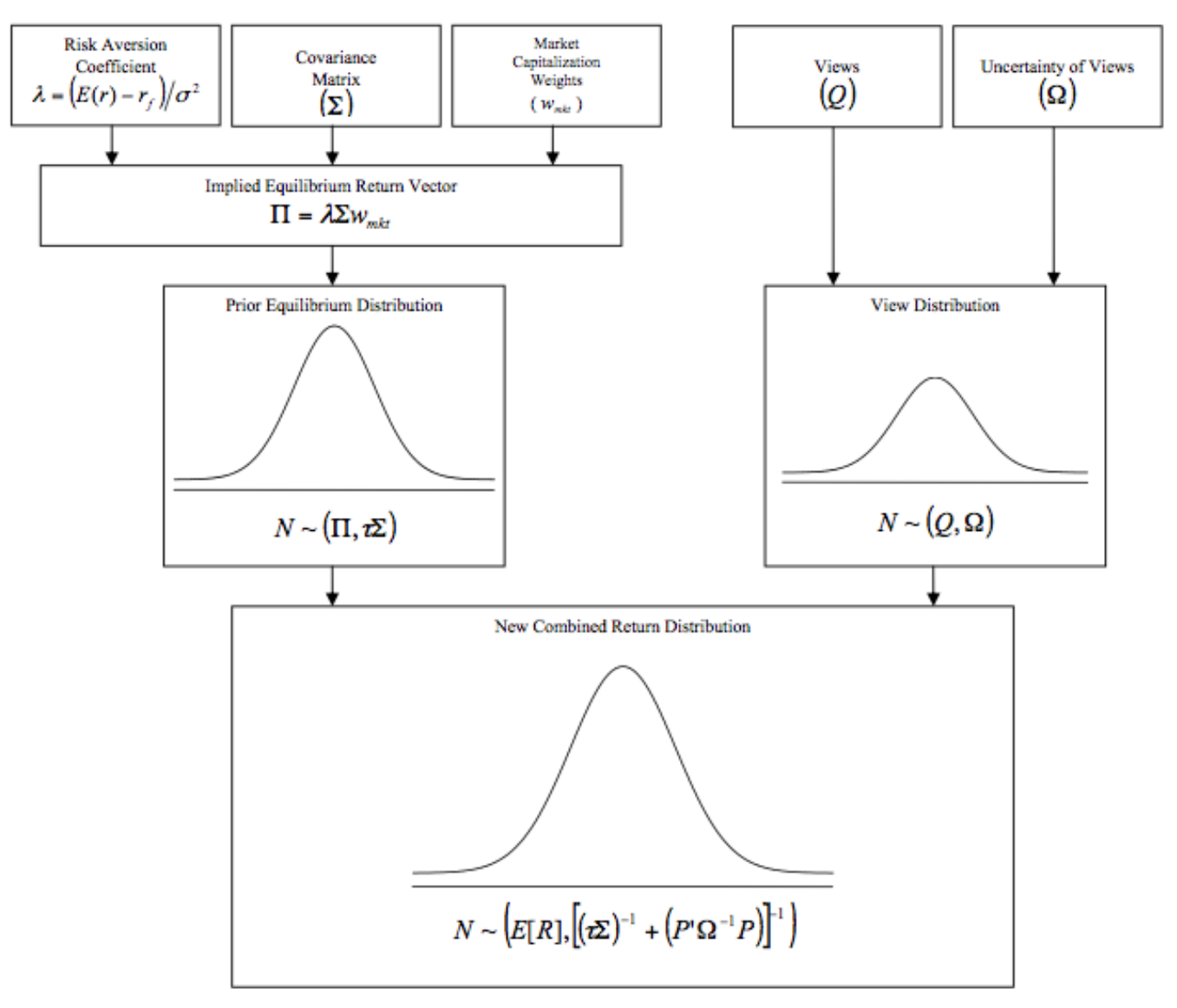




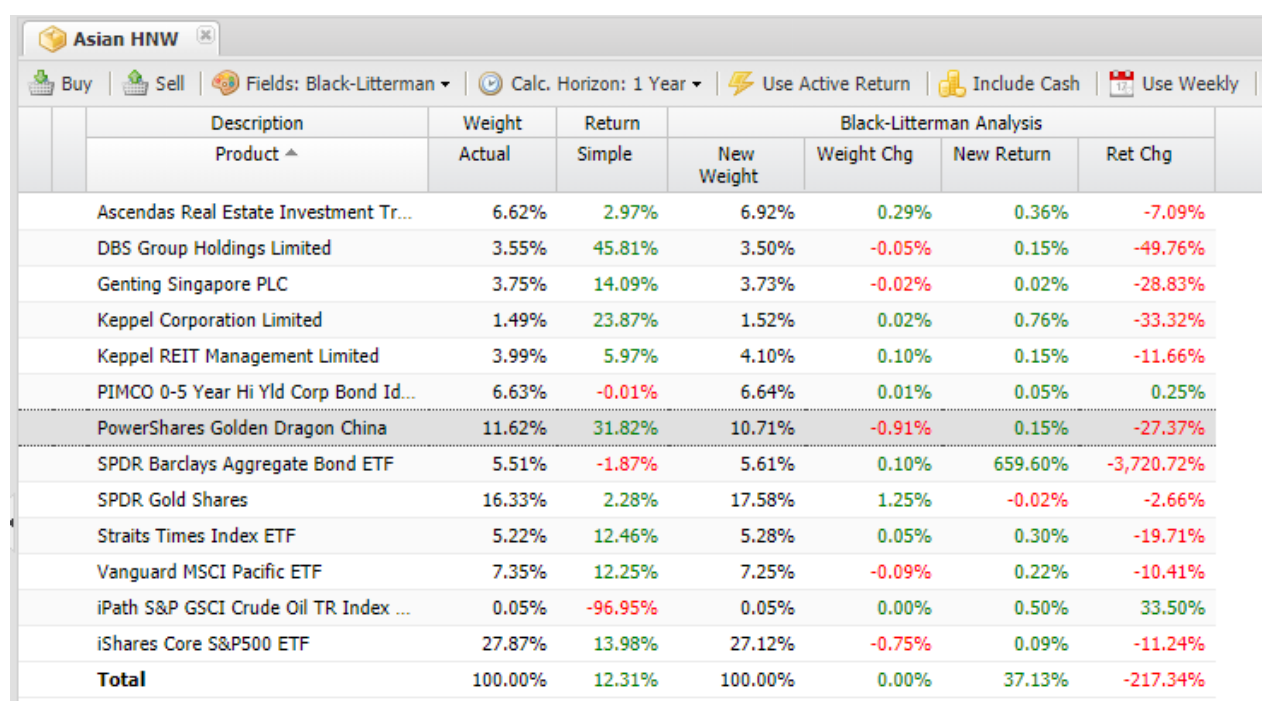

Figure 12.12: Sample Results from Stochastic Analysis

$P$ : The $P$ Matrix describes an investor's views. Each column of matrix $\mathrm{P}$ represents an investor's view about the assets' weighted expected returns, whose elements sum up to 1 .

Table 12.5: $P$ Matrix

\begin{tabular}{|r|r|r|}
\hline View1 & View 2 & \multicolumn{1}{|c|}{ View3 } \\
\hline 0.0132 & 0.1080 & 0.1985 \\
\hline 0.0492 & 0.0199 & 0.0317 \\
\hline 0.0237 & 0.0107 & 0.0187 \\
\hline 0.0000 & 0.0044 & 0.0057 \\
\hline 0.0785 & 0.1033 & 0.2067 \\
\hline 0.0426 & 0.0460 & 0.0342 \\
\hline 0.3784 & 0.0549 & -0.2701 \\
\hline-0.0511 & 0.0434 & 0.0033 \\
\hline 0.1466 & 0.1969 & 0.3987 \\
\hline-0.5885 & 0.0061 & 0.1351 \\
\hline 0.0388 & 0.0435 & 0.0871 \\
\hline 0.1798 & 0.1041 & 0.1050 \\
\hline 0.6887 & 0.2588 & 0.0456 \\
\hline
\end{tabular}


$Q:$ A column vector, in which an element represents a total change of a portfolio's weighted expected returns under a particular view.

Table 12.6: $Q$ Matrix

\begin{tabular}{|l|l|l|}
\hline View1 & View 2 & View3 \\
\hline $10.57 \%$ & $65.72 \%$ & $-12.29 \%$ \\
\hline
\end{tabular}

Diagonalized $(\Omega)$ Matrix : Diagonal covariance matrix of error terms describing the uncertainty in each view.

Table 12.7: Diagonalized $\Omega$ Matrix

\begin{tabular}{|c|c|c|}
\hline View1 & View 2 & View3 \\
\hline 0.0023 & 0.0000 & 0.0000 \\
\hline 0.0000 & 0.0006 & 0.0000 \\
\hline 0.0000 & 0.0000 & 0.0009 \\
\hline
\end{tabular}

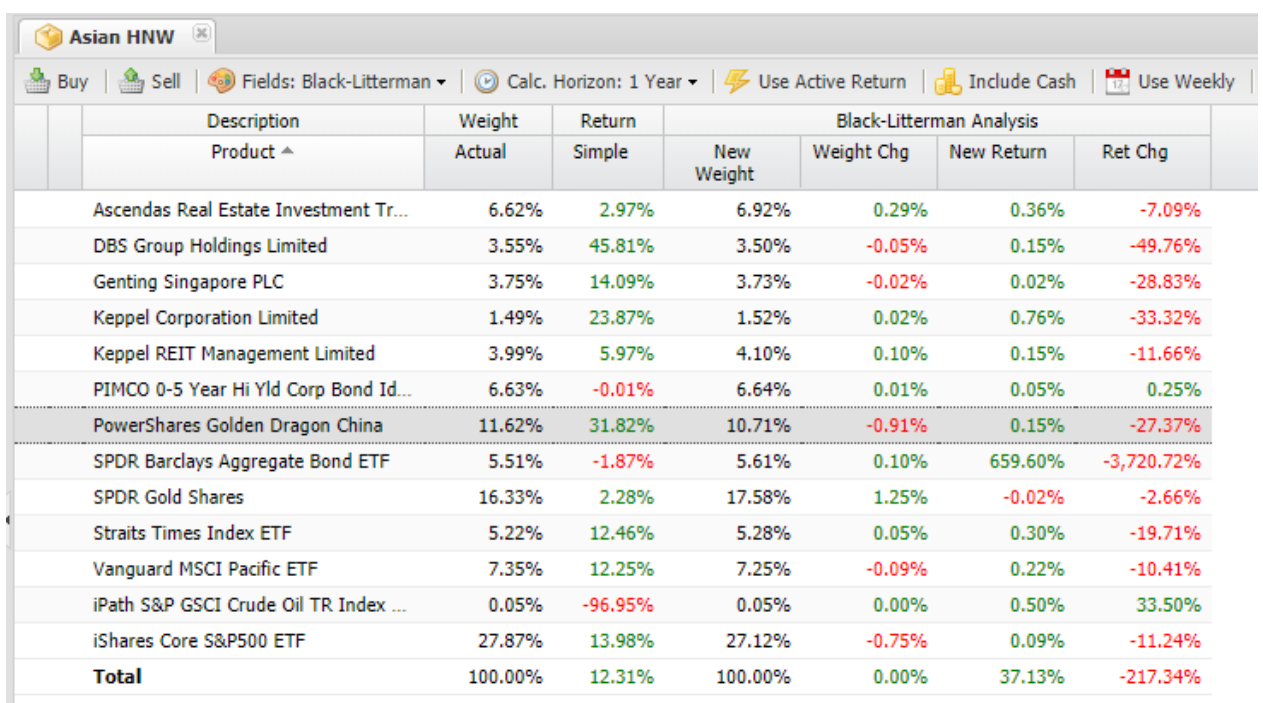

Figure 12.13: Results from the HedgeSPA Platform

(d) Finally, we will compare the calculation results between a stochastic analysis done semi-manually using a spreadsheet (likely to result in more significant 
numerical errors by the nature of any spreadsheet calculations) and computational results from the HedgeSPA platform using professional computational libraries are written in $\mathrm{C}++$, in order to illustrate the rough size of the numerical errors observed.

On the HedgeSPA platform, the stochastic analysis model is given by the following table. They are comparable, but there are noticeable numerical errors:

$$
E(R)=\Pi+\tau \Sigma P^{T}\left[\Omega+\tau P \Sigma P^{T}\right]^{-1}(Q-P \Pi)
$$

Assuming we are doing a comparison against a spreadsheet: First, we should calculate $\tau \Sigma P^{T}$, and then we need to compute $\left[\Omega+\tau P \Sigma P^{T}\right]^{-1}$, and finally $\tau \Sigma P^{T}\left[\Omega+\tau P \Sigma P^{T}\right]^{-1}$, as follows:

Table 12.8: Computations of $\tau \Sigma P^{T}$

\begin{tabular}{|r|r|r|}
\hline 0.0199 & 0.0155 & 0.0154 \\
\hline 0.0527 & 0.0289 & 0.0132 \\
\hline 0.0467 & 0.0273 & 0.0136 \\
\hline 0.0668 & 0.0357 & -0.0025 \\
\hline 0.0309 & 0.0183 & 0.0106 \\
\hline 0.0106 & 0.0069 & 0.0054 \\
\hline 0.0638 & 0.0240 & -0.0179 \\
\hline 0.0033 & 0.0019 & 0.0001 \\
\hline-0.0124 & -0.0008 & 0.0149 \\
\hline 0.0196 & 0.0120 & 0.0002 \\
\hline-0.0007 & 0.0028 & 0.0108 \\
\hline 0.0382 & 0.0217 & 0.0109 \\
\hline 0.0319 & 0.0165 & -0.0005 \\
\hline
\end{tabular}

Table 12.9: Computations of $\left[\Omega+\tau P \Sigma P^{T}\right]^{-1}$

\begin{tabular}{|r|r|r|}
\hline 270.1850 & -234.5155 & 45.6034 \\
\hline-234.5155 & 1019.1116 & -186.3331 \\
\hline 45.6034 & -186.3331 & 560.4212 \\
\hline
\end{tabular}


Table 12.10: Computations of $\tau \Sigma P^{T}\left[\Omega+\tau P \Sigma P^{T}\right]^{-1}$

\begin{tabular}{|r|r|r|}
\hline 0.1219 & 0.4153 & 0.3317 \\
\hline 0.4039 & 0.7292 & 0.2219 \\
\hline 0.3411 & 0.7174 & 0.2342 \\
\hline 0.4776 & 1.0605 & -0.2499 \\
\hline 0.2268 & 0.4718 & 0.1974 \\
\hline 0.0741 & 0.1787 & 0.1105 \\
\hline 0.5400 & 0.6401 & -0.5807 \\
\hline 0.0235 & 0.0547 & -0.0080 \\
\hline-0.1232 & -0.0360 & 0.3982 \\
\hline 0.1250 & 0.3777 & -0.0603 \\
\hline-0.0178 & 0.0495 & 0.2762 \\
\hline 0.2856 & 0.5582 & 0.1910 \\
\hline 0.2359 & 0.4721 & -0.0959 \\
\hline
\end{tabular}

Table 12.11: Computations of Posterior Returns $E(R)$

\begin{tabular}{|l|r|}
\hline Assets & Posterior returns \\
\hline Ascendas Real Estate Investment Trust & $0.7 \%$ \\
\hline DBS Group Holdings Limited & $1 \%$ \\
\hline Genting Singapore PLC & $1 \%$ \\
\hline Keppel Corporation Limited & $2 \%$ \\
\hline Keppel REIT Management Limited & $0.8 \%$ \\
\hline PIMCO 0-5 Year Hi Yld Corp Bond Idx ETF & $0.2 \%$ \\
\hline PowerShares Golden Dragon China & $2 \%$ \\
\hline SPDR Barclays Aggregate Bond ETF & $0.0 \%$ \\
\hline SPDR Dow Jones Global Real Estate & $1 \%$ \\
\hline SPDR Gold Shares & $0.1 \%$ \\
\hline Straits Times Index ETF & $1 \%$ \\
\hline Vanguard MSCI Pacific ETF & $1.2 \%$ \\
\hline iShares Core S\&P500 ETF & $1 \%$ \\
\hline
\end{tabular}


Table 12.12: Spreadsheet versus HedgeSPA Platform Results

\begin{tabular}{|r|r|}
\hline Posterior Weights from Spreadsheet & HedgeSPA Platform results \\
\hline $6 \%$ & $6 \%$ \\
\hline $2 \%$ & $2 \%$ \\
\hline $2 \%$ & $2 \%$ \\
\hline $2 \%$ & $1 \%$ \\
\hline $4 \%$ & $4 \%$ \\
\hline $6 \%$ & $6 \%$ \\
\hline $6 \%$ & $6 \%$ \\
\hline $11 \%$ & $11 \%$ \\
\hline $15 \%$ & $16 \%$ \\
\hline $16 \%$ & $15 \%$ \\
\hline $4 \%$ & $4 \%$ \\
\hline $5 \%$ & $5 \%$ \\
\hline $20 \%$ & $21 \%$ \\
\hline $100 \%$ & $100 \%$ \\
\hline &
\end{tabular}

\subsection{Conclusion}

Investors may face many known limitations and challenges when they try to generate a steady absolute return during the medium-to-long term. The Stochastic Analysis process is a solid choice to address these challenges, because the process can overcome the disadvantages of the traditional single-scenario approach, and provides an accurate estimation of a maximizing portfolio solution under multiple scenarios. Hence, the Stochastic Analysis tool on the HedgeSPA core investment platform can help investors to achieve a stable and profitable medium-to-long term investment. 